\title{
Synthetic Aperture Processing for Passive Co-prime Linear Sensor Arrays
}

\author{
Juan Ramirez Jr. Jeffrey L. Krolik \\ Duke Univeristy, Electrical and Computer Engineering, Durham, NC, 27708
}

\begin{abstract}
In this paper, we explore the use of synthetic aperture processing for optimizing the spatial covariance estimation capabilities of a moving linear co-prime sensor array. The linear co-prime sensor array geometry is a thinned linear array that is constructed by nesting uniform linear arrays with inter-related element spacing factors. The application of synthetic aperture processing in this setting is designed to create virtual sensors at missing half-wavelength intervals up to the degree required to produce a hole-free difference co-array across the full aperture of the synthetic array. Once a full set of spatial covariances are estimated covariance matrix based array processing methods can be applied as if the synthetic array were a uniform linear array. In this sense, the synthetic array is designed to approximate a uniform linear array while it retains a thinned linear array structure. We show simulation results examining the quality of the uniform linear array approximation afforded by this application synthetic aperture processing.
\end{abstract}

Keywords: Co-prime Sensor Arrays, Co-array Processing, Synthetic Aperture Methods.

\section{Introduction}

Thinning a sensor array is known to lead to severe sensor array processing deficiencies. These deficiencies manifest themselves in the array pattern

Email address: $\{$ j.ramirez,jlk\}@duke.edu (Juan Ramirez Jr. Jeffrey L. Krolik )

Preprint submitted to Digital Signal Processing, SI: CSA

July 27, 2016

(C) 2016. This manuscript version is made available under the Elsevier user license http://www.elsevier.com/open-access/userlicense/1.0/ 
4 specific to the sensor array geometry and can include periodic grating lobes, high-amplitude irregular side-lobes, as well as a loss in array gain. The severity by which these deficiencies occur is a function of the degree to which sensors are removed from the sensor array. The concept of removing sensors from an array and developing algorithms to overcome processing deficiencies has been a problem of interest in the thinned array literature [1, 2, 3]. Work in this area has been done to characterize and optimize the array patterns of thinned arrays in-terms of achieving desired sidelobe levels.

An equally important consequence of removing sensors from a sensor array can be seen via the spatial covariance estimation capability of the thinned array. Depending on the sensor placement the set of half-wavelength spatial covariances that can be estimated from sensor data can be incomplete. This leads to processing losses for signal-noise subspace and adaptive based array processing techniques. The well known Minimally Redundant Linear Array (MRLA) is an example of a thinned sensor array that preserves the half-wavelength spatial covariance sensing capabilities of the array over the entire array aperture [4. The MRLA is however computationally difficult to design for large apertures [5].

In the work presented here, we use array motion to expand the spatial covariance sensing capabilities of a thinned sensor array. In particular, we show how Synthetic Aperture (SA) processing can be used to extend the spatial covariance lag range of a sensor array having inter-element spacings on average larger than half-wavelength. This process will provide a synthetic array with an increase in the degrees of freedom available from each synthetic array snapshot, while not requiring all missing half-wavelength sensors to be synthesized during the SA process. The benefit of SA processing in this setting is a direct consequence of the physical array geometry. The physical array geometry considered in this work is based on the notion of nesting Uniform Linear Arrays (ULA) with related inter-element spacings. In recent literature, a sampling strategy based on this type of nesting of ULA's has been studied in the setting of source localization where the number of sources to be detected is greater than the 
number of sensors in the array [6]. In this work, the concept of the difference co-array was used to motivate the development and demonstrate the benefits of the nested array geometry concept. The difference co-array for a given sensor array characterizes the spatial covariances that can be estimated from the sensor array data. Here the difference co-array was of primary interest as it describes the degrees of freedom afforded by its underlying sensor array. It was shown that by applying the prescribed ULA nesting strategy an increase in the degrees of freedom could be realized from $\mathcal{O}(N)$ to $\mathcal{O}\left(N^{2}\right)$, where $N$ is the number of sensors in the array. In particular, it was shown that from this thinned sensor array geometry the spatial covariance lag range that can be estimated is equivalent to that of a ULA over the same aperture. While the spatial covariance lag coverage is equivalent to that of a ULA, the nested array geometry is suboptimal in the sensor count when compared to the MRLA. However, the simplicity in designing a nested array for large apertures provides an attractive trade-off in sensor count.

The array geometry of interest in this research is known as the linear Coprime sensor array. The co-prime sensor array concept is the result of relaxing the spatial covariance lag coverage requirements of the ULA nested array geometry. A linear co-prime sensor array is formed by nesting two ULA's with sensor spacings given by $M \frac{\lambda}{2}$ and $N \frac{\lambda}{2}$, respectively. The inter-element scale factors $M$ and $N$ are co-prime integers, meaning that the greatest common divisor of the pair of integers is unity. The co-prime sensing strategy has been developed for both temporal and spatial signals [7]. In particular, for sampling spatial signals, Pal and Vaidyanathan have shown that it is possible to form a contiguous set of $M N-1$ autocorrelation lags when $M$ and $N$ are co-prime and one of the subarrays is extended by a factor of two. It has been shown that this geometry is capable of providing $\mathcal{O}(M N)$ degrees of freedom from $\mathcal{O}(M+N)$ array elements. From this result, the co-prime array geometry can be used to approximate the sensing capabilities of a ULA with aperture $(M N-1) \frac{\lambda}{2}$. Even though it is possible to approximate a ULA with more sensors than are physically placed in the sensing aperture, the aperture of the equivalent ULA is about half of 


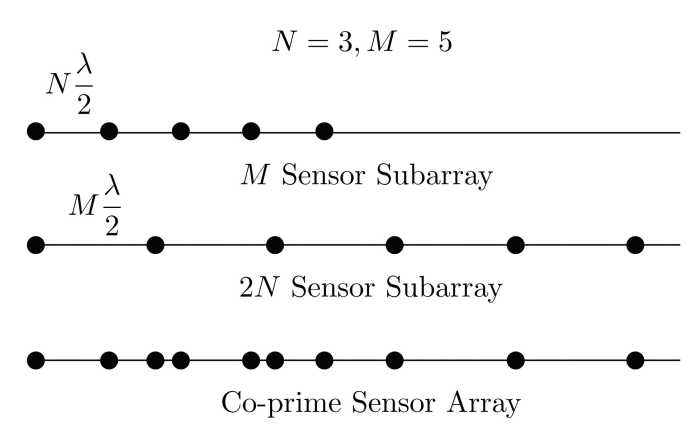

Figure 1: $N=3, M=5$ Co-prime Sensor Geometry

the physical co-prime array aperture. That is, the virtual ULA has aperture $(M N-1) \frac{\lambda}{2}$, while the physical aperture of the co-prime array is $(2 N-1) M \frac{\lambda}{2}$. Figure 1 shows an example of the above described co-prime geometry.

Filling missing elements of the difference co-array for co-prime arrays has also been considered in the situation where the co-prime array is on a non-moving platform. BauDaher et al. have developed a strategy for filling in difference coarray elements based on the use of related source frequencies [8]. Here the related source frequencies are used to realize the phases of virtual sensor elements that can be used to augment the difference co-array for a co-prime array.

\footnotetext{
The focus of this paper is to address the performance gap in estimating spatial covariances inherent to the co-prime array. In particular, we wish to estimate a full set of spatial covariances over the complete aperture of the coprime array. It has been shown that in situations where the array undergoes motion it is possible to increase the operational frequency of the array [9] [10]. Functionally this is a result of using physical sensors to sample at desired spatial locations, in the trajectory of the sensor array, to therefore virtually augment the sensor array up to the desired operational frequency. Adapting this concept, in this work we apply synthetic aperture processing to the co-prime sensor array in order to expand the spatial covariance sensing capability of the array. Here the objective is to virtually create missing sensor elements up to the degree required to produce a full set of spatial covariances over the aperture of the physical array
} 
and the additional aperture synthesized during the SA process. In particular, we propose an SA procedure that is a function of the co-prime parameters $N$ and $M$. The advantage of applying SA methods on a co-prime array is that the co-prime geometry guarantees half the physical aperture worth of spatial covariances and therefore with limited SA processing the missing spatial covariances can be realized.

In section 2 we review related co-prime and synthetic aperture work. Section 3 describes signal model and sensing environment and in section 4 we describe the synthetic aperture process for sensor synthesis and its connection to the difference co-array. In section 5 we propose our minimum temporal coherence criteria and describe its connection to the co-prime inter-element parameters $N$ and $M$. In section 6 we discuss spatial covariance matrix estimation methods and in section 7 we provide simulation examples demonstrating the benefits of SA for co-prime sensor arrays. Finally in section 8 we provide a discussion and conclusion of our primary results.

\section{Related Work}

The primary advantage of the co-prime geometry is its simplicity in design, while providing the degrees of freedom superior to the number of sensors used to form the array. A critical challenge in achieving the performance gains afforded by co-prime arrays is how to effectively estimate a spatial covariance matrix from the spatial covariance estimates that are obtained from the array sensor channel readings. Common problems encountered are rank deficiencies and lack of positive definiteness in the covariance matrix estimate.

Methods for estimating a spatial covariance matrix from thinned sensor array readings have been proposed over several decades. From the early works of Pillai and Haber to those of Abramovich et.al and more recently Pal and Vaidyanathan [11, 12, 6], to name a few. These techniques vary in approach, computational cost, and performance over different signal-to-noise-ratio regimes. The common objective of these methods is to produce a covariance matrix with 
dimension equal to the number of contiguous spatial covariances provided by the array. With a spatial covariance matrix estimate in hand one can apply array processing methods available as if the data were sensed on an equivalent ULA. The concept of ULA equivalence is based on the statistical equivalence between the thinned array snapshot and a ULA snapshot of which both can realize estimates of the same spatial covariances. In the work presented here, we apply three different spatial covariance matrix estimation techniques and explore their performance for source localization.

The novel contribution of this work is the non-standard application of SA processing. Typically, SA methods exploit platform motion to extend the physical aperture of an array and has lead to the vast work in Synthetic Aperture Radar (SAR) and SONAR. Synthetic aperture methods use temporal signal coherence to achieve improved array processing performance across the synthetic array aperture. Traditional SA techniques require temporal coherence that is proportional to the time required to travel the entire physical aperture. These methods have been used to improve angular resolution and signal gain over that provided by the physical array [13, 14, 15, 16].

While typically SA methods are used with uniformly spaced arrays, thinned array geometries have found applications in this setting. The MRLA has been used in SAR applications for the detection and localization of slow and fast moving targets [17. In this work, Zou et. al proposed a MRLA-SAR system that uses the non-uniform inter-elements spacings of the MRLA to improve the maximum velocity of a target that can be detected without ambiguity. In addition, the MRLA has been used in SONAR for aperture extension [18. Here the authors combine the well know Extended Towed Array Measurements (ETAM) algorithm for phase compensation with a fourth-order cumulant to extend the array aperture with fewer sensors than required when compared to a ULA. The work presented here continues this line of research but instead of aperture extension as the primary motivation for SA processing, the focus is on expanding the spatial covariance lag estimation range. Co-prime sensor arrays have been recently studied by the authors in the SA framework [19]. It was 
shown by using SA methods the achievable resolution of the co-prime array can be increased from the typical half-aperture resolution limit to the full extent of the physical aperture. In addition, as a function of the co-prime parameters, $N$ $M$, a SA strategy that produces a sensor array with a filled difference co-array has been developed [20]. The work presented here summarizes and expands upon these results as well as considers source localization performance when ideal sensing conditions are relaxed.

\section{Sensing Environment \& Signal Model}

The primary focus of this research is to develop an SA algorithm to estimate the spatial covariates of a ULA over a given aperture, but with less sensors than a ULA. In this situation, we consider a simplistic signal environment where a sensor array is towed with constant velocity with no perturbations from the medium. In addition, signal multi-path between source and receiver are not considered. These simplifications allow us to consider the SA algorithm without added complications. However, perturbations to the virtual sensors are considered in section 7. In future work, these factors will be studied in more detail with real data experiments.

Consider a co-prime array with a physical aperture of $L$ meters composed of $Q=M+2 N-1$ sensors. Let $d_{k}=\gamma_{k} \frac{\lambda_{0}}{2}$, with $k \in\{1, \cdots, Q\}$ be the sensor spacing measured relative to the left most sensor. The shift factors $\gamma_{k}$ are positive integers representing the integral number of half-wavelength spacings between sensor elements. Assume the array moves with a constant velocity $v$ along a straight-line course and that $K$ static incoherent radiating sources with common frequency $\Omega_{0}$ and bearings $\theta_{1}, \cdots, \theta_{K}$ are in the far-field of the array. In the work presented in this paper it is assumed that the source frequency $\Omega_{0}$ and platform velocity $v \mathrm{~m} / \mathrm{s}$ are known. Under these assumptions, the wave-field can be modeled as the sum of plane-waves.

Let the plane-wave signal received at the array from the $k$-th source be given 
by, $\mathbf{s}_{k}(t)=\alpha_{k} \exp (j \Omega t) \mathbf{b}\left(\theta_{k} ; \Omega\right)$ where $\alpha_{k} \sim \mathcal{C N}\left(0, \sigma_{k}^{2}\right)$, and

$$
\mathbf{b}\left(\theta_{k} ; \Omega\right)=\left[1, \exp \left(-j \Omega \frac{d_{1} \sin \left(\theta_{k}\right)}{c}\right), \cdots, \exp \left(-j \Omega \frac{d_{Q-1} \sin \left(\theta_{k}\right)}{c}\right)\right]^{\top}
$$

is the array manifold vector that encodes to co-prime array geometry. The factor $\alpha_{k}$ is a variable used to model the coherence time of the wave propogation medium and is further discussed in section 4.1. The frequency $\Omega$ includes the Doppler shift resulting from the relative motion between the source and the array given by $\Omega=\Omega_{0}\left(1-\frac{v \sin (\theta)}{c}\right)$. The Doppler shift frequency, $\Omega$, and array element spacing terms, $d_{l}$ with $l \in\{0,1, \cdots, Q-1\}$, in eq 1 , represent a sourcefrequency-element-spacing mismatch. It is well known when the array interelement spacing and frequency being sensed are not equivalent, $\lambda=\frac{c}{f}$, the array pattern is impacted by a widening/narrowing of the mainlobe. In addition, grating lobes appear when the source frequency is greater then the operational frequency of the array. Under certain conditions this mismatch can be negligible, to determine this condition we consider the argument of the array manifold vector described in eq 1. The argument of the $l$-th array manifold vector is given by,

$$
\begin{aligned}
\Omega \frac{d_{l} \sin \left(\theta_{k}\right)}{c} & =\Omega_{0}\left(1-\frac{v \sin \left(\theta_{k}\right)}{c}\right) \frac{d_{l} \sin \left(\theta_{k}\right)}{c} \\
& =\Omega_{0} \frac{d_{l} \sin \left(\theta_{k}\right)}{c}-\Omega_{0} d_{l} \frac{v \sin ^{2}\left(\theta_{k}\right)}{c^{2}}
\end{aligned}
$$

To enable an approximation the removes the source-frequency-element spacing mismatch from the array manifold vector model we require $\Omega_{0} d_{l} \frac{v \sin ^{2}\left(\theta_{k}\right)}{c^{2}} \approx 0$, expanding this term we have,

$$
\begin{aligned}
\Omega_{0} d_{l} \frac{v \sin ^{2}\left(\theta_{k}\right)}{c^{2}}= & \frac{c}{\lambda_{0}} \gamma_{l} \frac{\lambda_{0}}{2} \frac{v \sin ^{2}\left(\theta_{k}\right)}{c^{2}} \\
= & \gamma_{l} \frac{1}{2} \frac{v \sin ^{2}\left(\theta_{k}\right)}{c} \\
& <\gamma_{l} \frac{1}{2} \frac{v}{c}
\end{aligned}
$$

The final inequality in eq 3 suggests that when $v<<c$, the argument of the array manifold vector terms reduces to that of a matched source-frequencyelement spacing array. In addition, the dependance on the source angle $\theta_{k}$ on 
the Doppler shift frequency vanishes under this assumption. When $v<<c$, the $k$-th received signal is,

$$
\begin{gathered}
\mathbf{s}_{k}(t) \quad \alpha_{k} \exp (j \Omega t) \mathbf{b}\left(\theta_{k} ; \Omega_{0}\right) \\
=\alpha_{k} \exp \left(j \Omega_{0} t\right) \exp \left(-j \Omega_{0} \frac{v t \sin \left(\theta_{k}\right)}{c}\right) \mathbf{b}\left(\theta_{k} ; \Omega_{0}\right)
\end{gathered}
$$

where the array manifold vector now only depends on the source frequency $\Omega_{0}$. The snapshot collected at the array at time $t$ from a $K$-source field is given by,

$$
\mathbf{x}_{\mathrm{cp}}(t)=\sum_{l=1}^{K} \mathbf{s}_{l}(t)+\mathbf{w}(t)
$$

where $\mathbf{w}(t)$ is the receive noise vector is zero-mean complex additive Gaussian Noise with covariance $\sigma_{w}^{2} \mathbf{I}_{\mathbf{M}}, \mathbf{w}(t) \sim \mathcal{C N}\left(0, \sigma_{w}^{2} \mathbf{I}_{Q}\right)$. The probabilistic distribution of $\mathbf{x}_{\mathrm{cp}}(t)$ is complex Gaussian with mean and covariance given by,

$$
\begin{array}{cc}
\mathbf{E}\left(\mathbf{x}_{\mathrm{cp}}(t)\right) & =\mathbf{0} \\
\mathbf{E}\left(\mathbf{x}_{\mathrm{cp}}(t) \mathbf{x}_{\mathrm{cp}}(t)^{H}\right) & =\mathbf{B} \boldsymbol{\Lambda} \mathbf{B}^{H}+\sigma_{w}^{2} \mathbf{I}_{Q \times Q}
\end{array}
$$

where $\mathbf{B}=\left[\mathbf{b}\left(\theta_{1} ; \Omega_{0}\right) \mathbf{b}\left(\theta_{2} ; \Omega_{0}\right) \cdots \mathbf{b}\left(\theta_{k} ; \Omega_{0}\right)\right]$ encodes the directions of arrival of the signals received at the co-prime array and $\boldsymbol{\Lambda}=\operatorname{Diag}\left(\sigma_{1}^{2}, \cdots, \sigma_{k}^{2}\right)$ encodes the corresponding signal powers.

\section{Joint Sensor-Covariance Domain Augmentation}

Array motion allows for the increase of the dimension of the array manifold vector leading to an increase in the degrees of freedom of the array [6]. Let $\tau$ be the time for the array to travel a distance of $\frac{\lambda}{2}$ meters; then a synthetic array snapshot can be formed by appropriately interleaving the co-prime array snapshots from time instants $t, t+\tau, \cdots, t+\eta_{0} \tau$. The $t+l \tau$ portion of the synthetic snapshot, for $l \in\left\{1, \cdots, \eta_{0}\right\}$ is given by,

$$
\mathbf{s}_{l, k}(t)=\exp \left(-j \Psi_{l}\right) \mathbf{s}_{k}(t+l \tau)
$$

where $\Psi_{l}$ is a phase correction factor required to time-align the array snapshot from time $t+l \tau$ to time $t$. By evaluating $\mathbf{s}_{k}(t)$, defined above, at the augmentation instant $t+l \tau$, we see that 


$$
\begin{array}{r}
\mathbf{s}_{k}(t+l \tau)=\alpha_{k} \exp \left(j \Omega_{0} l \tau\right) \exp \left(j\left(\Omega_{0} t\right)\right) . \\
\exp \left(-j \Omega_{0} \frac{v t \sin \left(\theta_{k}\right)}{c}\right) \cdot \exp \left(-j \Omega_{0} \frac{v l \tau \sin \left(\theta_{k}\right)}{c}\right) \mathbf{b}\left(\theta_{k} ; \Omega_{0}\right)
\end{array}
$$

From this expression, we see that the phase correction factor is given by $\Psi_{l}=\Omega_{0} l \tau$. Since $v l \tau=l \frac{\lambda}{2}$, elements of the synthetic array manifold vector are formed by the product $\exp \left(-j \Omega_{0} \frac{v l \tau \sin \left(\theta_{k}\right)}{c}\right) \mathbf{b}\left(\theta_{k}\right)$. Let $\tilde{\mathbf{b}}\left(\theta_{k} ; \Omega_{0}\right)$ represent a component of the synthetic array manifold vector given by,

$$
\begin{array}{r}
\tilde{\mathbf{b}}\left(\theta_{k} ; \Omega_{0}\right) \quad=\exp \left(-j \Omega_{0} l \frac{\lambda}{2} \frac{\sin \left(\theta_{k}\right)}{c}\right) \mathbf{b}\left(\theta_{k} ; \Omega_{0}\right) \\
=\quad \exp \left(-j \Omega_{0} l \frac{\lambda}{2} \frac{\sin \left(\theta_{k}\right)}{c}\right), \exp \left(-j \Omega_{0} \frac{\left(\gamma_{1}+l\right) \frac{\lambda}{2} \sin \left(\theta_{k}\right)}{c}\right), \\
\left.\cdots, \exp \left(-j \Omega_{0} \frac{\left(\gamma_{M}+l\right) \frac{\lambda}{2} \sin \left(\theta_{k}\right)}{c}\right)\right]^{\top}
\end{array}
$$

with half-wavelength sensor position shifts $\gamma_{1^{\prime}}(l \tau)=\gamma_{1}+l, \gamma_{2^{\prime}}(l \tau)=\gamma_{2}+$ $l, \cdots, \gamma_{M^{\prime}}(l \tau)=\gamma_{M}+l$. By interleaving the physical and synthetic snapshot vectors, the augmented signal received at the array is given by,

$$
\mathbf{s}_{k}^{\prime}(t)=\mathbf{T}_{0} \mathbf{s}_{k}(t)+\sum_{l=1}^{\eta_{0}} \exp \left(-j \Psi_{l}\right) \mathbf{T}_{l} \mathbf{s}_{k}(t+l \tau)
$$

where $\mathbf{T}_{i}: \mathcal{C}^{Q} \rightarrow \mathcal{C}^{Q^{\prime}}$ is 1's and 0's selection matrix that extends the $Q$ dimensional physical snapshot to a $Q^{\prime}$-dimensional snapshot vector. The product $\exp \left(-j \Psi_{l}\right) T_{l} \mathbf{s}_{k}(t+l \tau)$ produces a time-aligned vector of length $Q^{\prime}$ that inserts the entries of $\mathbf{s}_{k}(t+l \tau)$ in the appropriate synthesis positions. The snapshot vector $\mathbf{s}_{k}^{\prime}(t)$ represents a wave-field sensed at an array with $Q^{\prime}$ sensors, with sensor spacing given by $\tilde{d}_{i}=\beta_{i} \frac{\lambda}{2}$ with,

$$
\beta_{i} \in\left\{\gamma_{m}\right\}_{m=1}^{Q} \bigcup_{l=1}^{L}\left\{\gamma_{m^{\prime}}(l \tau)\right\}_{m=1}^{Q}
$$

The subsequent snapshot collected at the synthetic array is given by,

$$
\mathbf{x}_{\mathrm{sa}}(t)=\sum_{l=1}^{K} \mathbf{s}_{l}^{\prime}(t)+\mathbf{w}^{\prime}(t)
$$

228 where $\mathbf{w}^{\prime}(t) \sim \mathcal{C N}\left(0, \sigma_{w}^{2} \mathbf{I}_{\mathbf{Q}^{\prime}}\right)$. The probabilistic distribution of the synthetic array snapshot is a complex Gaussian with mean and covariance, 


$$
\begin{array}{rc}
\mathbf{E}\left(\mathbf{x}_{\mathrm{sa}}(t)\right) & =\mathbf{0} \\
\mathbf{E}\left(\mathbf{x}_{\mathrm{sa}}(t) \mathbf{x}_{\mathrm{sa}}(t)^{H}\right) & =\tilde{\mathbf{B}} \boldsymbol{\Lambda} \tilde{\mathbf{B}}^{H}+\sigma_{w}^{2} \mathbf{I}_{Q^{\prime} \times Q^{\prime}}
\end{array}
$$

where $\tilde{\mathbf{B}}=\left[\tilde{\mathbf{b}}\left(\theta_{1} ; \Omega_{0}\right) \tilde{\mathbf{b}}\left(\theta_{2} ; \Omega_{0}\right) \cdots \tilde{\mathbf{b}}\left(\theta_{k} ; \Omega_{0}\right)\right]$ encodes the directions of arrival of far-field signals received at the synthetic array and $\boldsymbol{\Lambda}=\operatorname{Diag}\left(\sigma_{1}^{2}, \cdots, \sigma_{k}^{2}\right)$ encodes the corresponding signal powers.

The sensor synthesis process increases both the number of "sensors" in the array and the array aperture. The sensor count increases since virtual sensors are inserted into the array after each synthesis step. The aperture of the array is increased by $\frac{\lambda}{2}$ meters after each synthesis step and after $\eta_{0}$ synthesis steps the aperture of the array is increased by $\eta_{0} \frac{\lambda}{2}$ meters. However, the number of "sensors" after synthesis is dependent on the initial sensor distribution of the array. This discrepancy is due to the possibility of sensor overlap between snapshots at different synthesis instants. This overlap can be advantageous as it can allow for estimation of the phase correction factor [21]. In the work presented here, the phase correction factor is assumed to be known.

\subsection{Coherent Space-Time Modeling}

To have spatial coherence across the synthetic array we require temporal coherence within the time window required to form the synthetic array snapshot. This can be captured in the above array motion signal model by letting $\alpha_{k}$, for each source, be constant, (i.e. coherent) for the time required for the array to travel a distance of $\eta_{0} \frac{\lambda}{2}$ meters. In this application of $\mathrm{SA}$, we require spatial coherence only over a fraction of the array aperture since we are interested in expanding the spatial covariance estimation capability of the array physical array. Since the physical array is co-prime it inherently has an extended range of spatial covariance lag coverage thereby requiring limited coherence time to realize all missing covariance estimates. Traditional SA techniques require coherence over much longer distances typically on the order of the array aperture. 
To model this requirement, we form one synthetic array snapshot every $n_{0} \tau+T_{s}$ seconds, where $T_{s}$ is the system sampling time, and associate an i.i.d. signal amplitude vector $\vec{\alpha}\left(t_{m}\right)=\left[\alpha_{1}, \cdots, \alpha_{k}\right]^{\top}$ for each snapshot interval. Each element of $\vec{\alpha}\left(t_{m}\right)$ is drawn $\sim \mathcal{C N}\left(0, \sigma_{k}^{2}\right)$. Under this coherence model we obtain a synthetic array snapshot where across the array the complex random amplitude is constant for each spatial signal present in the field. This model preserves the plane-wave structure of the wave-field over the aperture of the synthetic array snapshot.

\section{2. $v<<c$ Approximation Implications for SA Processing}

The SA process is centered on creating the array manifold components for missing sensors from appropriate time shifts of the received data. In this section, we consider the implications of the source-frequency-element spacing mismatch after the synthetic aperture process. Suppose the distance between the $l$-th and $l+1$-th sensors is $\lambda_{0}(\mathrm{~m})$ then SA can be used to produce a virtual sensor between the $l$-th and $l+1$-th physical sensors. In this case, the virtual sensor is produced by sampling channel data from the $l$-th sensor when it traverses a distance of $\frac{\lambda_{0}}{2}(\mathrm{~m})$. With $\tau$ representing the time required for the array to travel $\frac{\lambda_{0}}{2}(\mathrm{~m})$ we have virtual channel data derived from the SA process is given by,

$$
\begin{array}{r}
s_{l}(t+\tau)=\alpha_{k} \exp (j \Omega(t+\tau)) b_{l}(\theta ; \Omega) \\
=\alpha_{k} \exp (j \Omega t) \exp (j \Omega \tau) b_{l}(\theta ; \Omega) \\
=\alpha_{k} \exp (j \Omega t) \exp \left(j \Omega_{0}\left(1-\frac{v \sin (\theta)}{c}\right) \tau\right) b_{l}(\theta ; \Omega) \\
=\alpha_{k} \exp (j \Omega t) \exp \left(j \Omega_{0} \tau\right) \exp \left(-j \Omega_{0} \frac{v \tau \sin (\theta)}{c}\right) b_{l}(\theta ; \Omega)
\end{array}
$$

Here $\exp \left(j \Omega_{0} \tau\right)$ is the phase term that is corrected during the SA process. Here we consider the $l$-th component of the array manifold vector defined in eq 1 . The virtual component of the array manifold vector is derived from the product, $\exp \left(-j \Omega_{0} \frac{v \tau \sin (\theta)}{c}\right) b_{l}(\theta ; \Omega)$. Expanding this term and recognizing that $v \tau=\frac{\lambda_{0}}{2}$ we have that 


$$
\begin{array}{r}
\exp \left(-j \Omega_{0} \frac{v \tau \sin (\theta)}{c}\right) b_{l}(\theta ; \Omega) \\
=\exp \left(-j \Omega_{0} \frac{\frac{\lambda_{0}}{2} \sin (\theta)}{c}\right) \exp \left(-j \Omega_{0} d_{l} \frac{\sin (\theta)}{c}\right) \exp \left(-j \Omega_{0} \frac{v \sin ^{2}(\theta)}{c^{2}} d_{l}\right) \\
=\exp \left(-j \Omega_{0}\left(\frac{\lambda_{0}}{2}+d_{l}\right) \frac{\sin (\theta)}{c}\right) \exp \left(-j \Omega_{0} \frac{v \sin ^{2}(\theta)}{c^{2}} d_{l}\right)
\end{array}
$$

The quantity $\frac{\lambda_{0}}{2}+d_{l}$ represents the distance off-set for a sensor between the $l$-th and $l+1$-th physical sensors. The term $\exp \left(-j \Omega_{0}\left(\frac{\lambda_{0}}{2}+d_{l}\right) \frac{\sin (\theta)}{c}\right)$ represents the phase shift of a sensor placed between the $l$-th and the $l+1$-th sensor for an array with operational wavelength $\lambda_{0}$ sensing a frequency $\Omega_{0}$. The term $\exp \left(-j \Omega_{0} \frac{v \sin ^{2}(\theta)}{c^{2}} d_{l}\right)$ is an arrival angle dependent phase offset which reduces to $\exp \left(-j \frac{v \sin ^{2}(\theta)}{c} \frac{l}{2}\right)$ since $\Omega_{0}=c / \lambda_{0}$ and $d_{l}=l \lambda_{0} / 2$. It is clear that the primary factors that influence this phase term is the ratio of tow velocity to sound propagation speed.

The array manifold vector after SA processing is given by,

$$
\tilde{\mathbf{b}}\left(\theta ; \Omega, \Omega_{0}\right)=\left[\begin{array}{c}
{[} \\
1 \\
\vdots \\
\exp \left(-j \Omega_{0}\left(\frac{\lambda_{0}}{2}+d_{l}\right) \frac{\sin (\theta)}{c}\right) \exp \left(-j \frac{v \sin ^{2}(\theta)}{c} \frac{l}{2}\right) \\
\exp \left(-j \Omega d_{l+1} \frac{\sin (\theta)}{c}\right) \\
\vdots \\
\exp \left(-j \Omega d_{Q-1} \frac{\sin (\theta)}{c}\right)
\end{array}\right]
$$

Here $\tilde{\mathbf{b}}\left(\theta ; \Omega, \Omega_{0}\right)$ has an additional sensor when compared to $\mathbf{b}(\theta ; \Omega)$, however the frequency of the SA processed channel does not match the Doppler shift frequency. The mismatch in phase delay over the aperture of the array deteriorates the spatial coherence of the synthetic array. The degree of degradation of the spatial coherence is a function of the ratio of the platform velocity to sound speed of the medium. This ratio will influence the Doppler Shift frequency mismatch and the phase offset introduced during the SA process. To measure the 
loss of spatial coherence over the array we consider the ratio of the area under the array pattern formed from an array with SA processing and a ULA with no SA processing. The area under the ULA array pattern serves as a reference for perfect spatial coherence, while the area under the SA array pattern captures the effects of phase offsets. This metric is defined as

$$
A=\frac{\int\left|\left\langle h, \mathbf{b}_{\mathrm{SA}}(\theta, \Omega)\right\rangle\right| d \theta}{\int\left|\left\langle h, \mathbf{b}_{\mathrm{ULA}}(\theta, \Omega)\right\rangle\right| d \theta}
$$

where $h$ is an all ones vector of length equal to the number of sensors in the array and $\mathbf{b}_{\mathrm{SA}}(\theta, \Omega)$ and $\mathbf{b}_{\mathrm{ULA}}(\theta, \Omega)$ are the array manifold vectors for the SA processed array and Uniform Line Array, respectively. When $A>1$ phase coherence on the synthetic array has deteriorated to the degree large side-lobe distortions are present in the corresponding array pattern when compared to the ULA.

We consider an array aperture of $26 \lambda_{0} / 2$, a ULA over this aperture consists of 27 sensors spaced $\lambda_{0} / 2(\mathrm{~m})$ apart. Suppose the sensor locations of this ULA are indexed $\{0,1,2, \cdots, 25,26\}$ and that the ULA will have virtual sensors at index positions $l \in\{1,3,6,10,12,15,20,25\}$. In this situation each of these virtual sensors contribute to the spatial coherence mismatch generated during the SA process. Figure 2 (a) shows the array pattern area ratio as a function of the $v / c$. Here it is shown that for $v / c<10^{-2}$, the area ratios are nearly unity. This implies that the impact of the spatial coherence mismatch discussed above produces little to no distortion in the array pattern of the synthetic array. Figure 2 (b) shows a sample array pattern. Here both the ULA and synthetic array patterns are shown. In perfect SA processing conditions, Figure 2 (b) shows that for 10 knots the loss of coherence produces a minor side-lobe increase. This measure implies that while the Doppler-frequency-element-spacing mismatch can impact the spatial coherence over the array, the $v<<c$ assumption allows for removing this mismatch from the signal model as done in eq 4 is justified. 


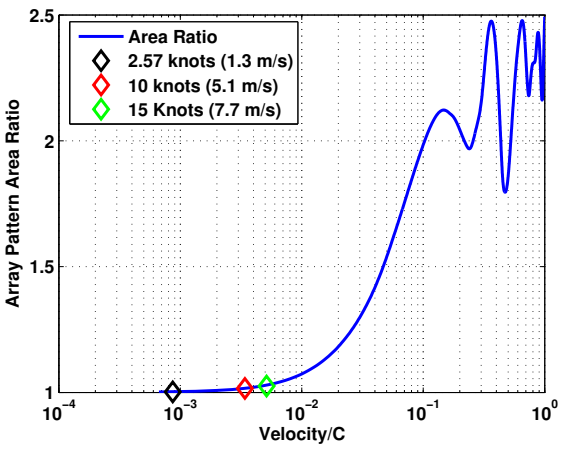

(a) Area Ratio

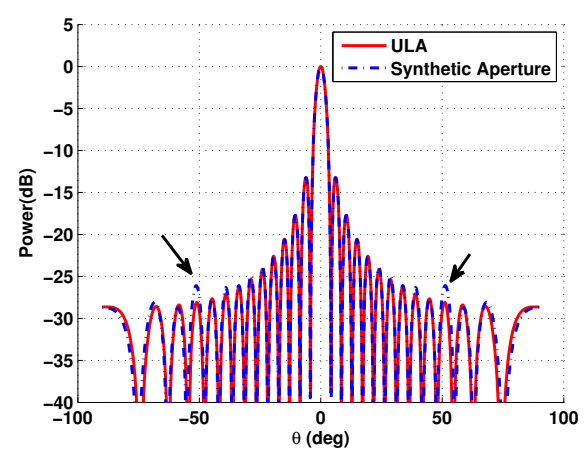

(b) Array Pattern: $v=10$ Knots

Figure 2: Area Under Array Pattern

\section{Minimum Temporal Coherence Period}

Our objective is to form a synthetic array snapshot for an array with denser sensor sampling than what is available from the co-prime array. Our main strategy is to exploit the motion of the array to form the denser snapshot. As described in detail above, when the co-prime array is moved along a straight line path, measurements from different times can be fused together to fill-in desired sensor spacings. In order to accomplish this we must consider the coherence time of the spatial signals and the noise measured at the array.

SA processing requires that the coherence time be longer than the time needed for the array to move the required distance to form a given snapshot. 
Let $C_{t}$ be the coherence time, then we need to satisfy,

$$
\frac{D}{v} \leq C_{t}
$$

where $D$ is to total distance traveled by the array during the synthetic aperture integration period and $v$ is the velocity the array system is moving. Our goal is to determine how to minimize the total distance traveled by the co-prime array in order to maximize the spatial covariance lag range available from the synthetic array snapshot.

The optimization criteria is to produce a full set of spatial covariance measurements from each synthetic array snapshot, while minimizing the total synthetic aperture integration time window. In addition, this optimization criteria can be formalized in terms of the co-prime inter-element pair $N, M$. That is to say, as a function of the co-prime geometry we can determine the minimum number of $\frac{\lambda}{2}$ synthetic aperture integration iterations needed to fill the difference co-array over the aperture of the co-prime array.

The degrees of freedom of a line array are related to the number of unique spatial covariances that can be observed from the array snapshot. For co-prime arrays, Pal and Vaidyanathan have shown the co-array has a hole-free contiguous region of approximately half of the array aperture [6] [7. This hole-free region is directly related to the degrees of freedom present in the array and is the primary reason the co-array plays a central role in SA methods 22. Ramirez Jr. et al. have developed an upper bound on the Temporal-Coherence-Period (TCP) required to produce a filled co-array without synthesizing every missing sensor over the array aperture in a moving co-prime sensor array system [19. This however, only provides a sufficient condition for filling the co-array. The necessary and sufficient condition for minimizing TCP while maximizing the degrees of freedom over the array aperture of a moving co-prime array has been formalized and is published in [20] and is restated here. The mathematical details are provided in [20.

Lemma 1. The minimum Temporal-Coherence-Period for a $(N, M) \lambda / 2$ coprime array with $M+2 N-1$ sensor elements and $N<M$ to have a hole-free 
co-array across the array aperture is given by $T C P=\eta_{0} \tau$ where,

$$
\eta_{0}= \begin{cases}\frac{N}{2} & \text { if } N \text { is even } \\ \frac{N-1}{2} & \text { if } N \text { is odd }\end{cases}
$$

and $\tau$ is the time required for the array to travel a distance of $\lambda / 2$ meters.

This Lemma suggests that the minimum TCP is a function of the co-prime factor $N$ and independent of the larger co-prime factor $M$. From this perspective, we can see that the minimum TCP is proportional to the time required for the array to travel $\frac{N}{2} \frac{\lambda}{2}(\mathrm{~m})$ of the physical aperture.

The significance of the minimum TCP is that it describes the minimum distance required for a co-prime sensor array to travel for SA methods to produce a filled co-array. The hole-free co-array over the array aperture provides a full set of spatial covariances required to maximize the degrees of freedom available from the array aperture needed for signal subspace based source localization. The advantage of the work presented here is that via SA methods we are able to maximize the degrees of freedom over a given aperture with minimum synthesis time.

Figure 3 shows examples of the difference co-array for various amounts of SA processing on a particular linear co-prime sensor array. Here we consider a co-prime sensor array with parameters $N=5, M=7$ with aperture $63 \frac{\lambda}{2}$, and the minimum number of SA iterations required to fill the difference co-array is $\eta_{0}=2$. In addition, the locations of the physical an virtual sensors are indicated at the top of each figure. From Figure 3 we see that at the above described minimum temporal coherence period we have a difference co-array that is hole free. In principle one could continue the SA process until the synthetic array has no missing sensors and produce the difference co-array corresponding to a ULA. However the price of this is an increase in the total SA process time to form one synthetic snapshot. The advantage of utilizing SA over the minimum coherence temporal period is that one has access to spatial covariance estimates from a thinned array as if these estimates were formed from a ULA. In principle, SA processing can be applied to any thinned array geometry to augment the 
Table 1: Co-prime Array Integration Time Lengths

\begin{tabular}{|c||c|c|}
\hline \multicolumn{3}{|c|}{$c=1500 \frac{m}{s}, v=1.3 \frac{m}{s}, f=440 \mathrm{~Hz}$} \\
\hline$N, M$ & Physical Aperture $(2 N-1) M \frac{\lambda}{2}(m)$ & $\frac{\eta_{0} \frac{\lambda}{2}}{v}(\mathrm{~s})$ \\
\hline 3,5 & $25 \frac{\lambda}{2}=42.61$ & 1.31 \\
4,9 & $63 \frac{\lambda}{2}=107.3$ & 2.62 \\
5,9 & $81 \frac{\lambda}{2}=138.0$ & 2.62 \\
6,11 & $121 \frac{\lambda}{2}=206.2$ & 3.93 \\
7,13 & $169 \frac{\lambda}{2}=288.0$ & 3.93 \\
8,15 & $225 \frac{\lambda}{2}=383.5$ & 5.24 \\
9,17 & $289 \frac{\lambda}{2}=492.6$ & 5.24 \\
\hline
\end{tabular}

difference co-array. In particular, a Minimum-Hole array can be designed and SA processing can be used to fill-in missing sensors such that the difference co-array becomes hole-free.

Table 1 and 2 show typical co-prime array geometries along with the time required to form one synthetic array snapshot in seconds for two different frequency regimes. In these situations minimum required temporal coherence to produce an synthetic array with a hole-free co-array is on the order of a few seconds. While these estimates represent the theoretical requirements to apply SA in our situations typical temporal coherence can range on the order of several to tens of minutes. Williams and Battestin have reported coherence times of 8-12 minutes at $400 \mathrm{~Hz}$, in the deep ocean 23, 24. Similarly, Yang has studied the temporal coherence of sound through shallow water over a wide range of frequencies [25]. In this work, Yang found coherence time estimates of between 5 and 20 minutes. While temporal coherence can vary widely over signal frequency and range the estimates found in the literature support the use of SA in our application. 
Table 2: Co-prime Array Integration Time Lengths

\begin{tabular}{|c||c|c|}
\hline \multicolumn{3}{|c|}{$c=1500 \frac{m}{s}, v=1.3 \frac{m}{s}, f=1 \mathrm{kHz}$} \\
\hline$N, M$ & Physical Aperture $(2 N-1) M \frac{\lambda}{2}(m)$ & $\frac{\eta_{0} \frac{\lambda}{2}}{v}(\mathrm{~s})$ \\
\hline 3,5 & $25 \frac{\lambda}{2}=18.75$ & 0.5769 \\
4,9 & $63 \frac{\lambda}{2}=47.25$ & 1.15 \\
5,9 & $81 \frac{\lambda}{2}=60.75$ & 1.15 \\
6,11 & $121 \frac{\lambda}{2}=90.75$ & 1.73 \\
7,13 & $169 \frac{\lambda}{2}=126.75$ & 1.73 \\
8,15 & $225 \frac{\lambda}{2}=168.75$ & 2.30 \\
9,17 & $289 \frac{\lambda}{2}=216.75$ & 2.30 \\
\hline
\end{tabular}

\section{Spatial Covariance Matrix Estimation}

From a synthetic array snapshot, our goal is to form a positive-definite spatial covariance matrix that can be used for subspace based source localization with characteristics equivalent to a ULA. The synthetic array constructed from a co-prime array with the minimum Temporal Coherence Period criteria is capable of estimating a full set of spatial covariances over the aperture of the array. However, the synthetic array remains a thinned sensor array over the composite synthetic aperture since all missing sensor elements from the co-prime array are not virtually formed during the SA process. This implies that an outer product of synthetic array snapshots will not produce a spatial covariance matrix with the desired ULA characteristics. Instead, the spatial covariances that can be estimated from the synthetic array must be manipulated to form the desired spatial covariance matrix that approximates the ULA over the same aperture. In this section, we consider three different methods for producing a spatial covariance matrix from sensor measurements, first is the Direct Augmentation Algorithm (DAA), second is a method based minimizing the projection of the DAA estimate onto the space of positive-definite matrices, and finally the Rank Enhanced Spatial Smoothing (RESS) algorithm proposed by Pal and 
Vaidyanathan.

Using the Direct Augmentation Algorithm (DAA), the co-array and the available spatial covariances provide an estimate of the $l$-th lag of the spatial covariance given by,

$$
\hat{R}_{x}(l, t)=\frac{1}{c(l)} \sum_{\substack{\left(m_{1}, m_{2}\right) \in \mathcal{V}(l) \\ m_{1} \leq m_{2}}}(\mathbf{x}(t))_{m_{1}}(\mathbf{x}(t))_{m_{2}}^{*}
$$

where $\mathcal{V}(l)$ is the set of array snapshot element index pairs whose difference is $l$. By collecting the samples into a Toeplitz matrix, we can obtain an estimate of the augmented spatial-covariance matrix. However, estimating the covariance matrix in this manner does not guarantee positive-definiteness and may lead to negative eigenvalues in the spatial covariance matrix estimate.

Abramovich et al. has proposed methods for Toeplitz Positive-Definite covariance matrix completion from the DAA spatial covariance matrix estimate [12. However, these methods can lead to unstable localization results and are computationally expensive. A more tractable approach was introduced by Kazanci and Krolik [26]. In this work, they developed a technique known as Adaptive Channel Compensation (ACC) for dealing with sensor arrays with faulty sensors within the array aperture. The main idea of this technique is to use the reliable sensors along with an estimate of the full array covariance matrix to produce an estimate of sensor readings at the missing sensors. In this technique an estimate of a hole-free sensor array snapshot is produced via an optimization procedure. We have explored applying this technique to overcome positive definite deficiency encountered from estimating the spatial covariance matrix using the Direct Augmentation Approach. The optimization problem can be formulated as the following: we seek to find an array snapshot $\hat{\mathbf{q}}$, that will produce an outer product that is "closest" to the DAA covariance matrix estimate of a single snapshot from the synthetic array, while minimizing its projection onto the subspace spanned by the eigenvectors corresponding to the negative eigenvalues of the DAA estimate. The array snapshot $\hat{\mathbf{q}}$ is a hole-free channel data vector over the aperture spanned by the synthetic array. This is 
formalized mathematically by,

$$
\hat{\mathbf{q}}=\min _{\mathbf{q}}|| \Phi_{\text {neg }}^{H} \mathbf{q} \|_{F}^{2}
$$

${ }_{447}$ subject to the constraint $\left\|\mathbf{L}^{H} \mathbf{q}-\mathbf{x}_{g}\right\|<\epsilon$.

Where $\hat{\mathbf{q}}$ is the hole-free array snapshot estimate, $\boldsymbol{\Phi}_{\text {neg }}$ characterizes the subspace spanned by the negative eigenvectors of the single snapshot DAA covariance matrix estimate, $\mathbf{x}_{g}$ is a vector of sensor readings from the synthetic array, $\mathbf{L}$ identifies the sensors in $\hat{\mathbf{q}}$ corresponding to the same channels in the synthetic array, and $\epsilon$ is a closeness tolerance on elements of the snapshot estimate that correspond to synthetic array sensor readings. From this point of view, the snapshot estimate $\hat{\mathbf{q}}$ preserves channel data corresponding to the synthetic array channels, while the optimization process produces estimates of channel data for the remaining channels of $\hat{\mathbf{q}}$. The closed form solution to this optimization problem can be found by an application of Lagrangian multipliers and is given by,

$$
\hat{\mathbf{q}}=\left(\boldsymbol{\Phi}_{\text {neg }} \boldsymbol{\Phi}_{\text {neg }}^{H}+\mu \mathbf{L} \mathbf{L}^{H}\right)^{-1} \mu \mathbf{L} \mathbf{x}_{g}
$$

where $\mu$ is a regularization parameter used to tune the optimization process. In this approach, we seek to find an array snapshot estimate $\hat{\mathbf{q}}$ that produces a spatial covariance matrix estimate that is the closest postive-definite matrix to the DAA covariance matrix estimate derived from a single synthetic array snapshot. For a given synthetic array snapshot we can produce a virtual array snapshot that has no missing sensors over the aperture of the array that can be used to produce a covariance matrix estimate by averaging outer-products. This modification to the ACC covariance estiamation method will be denoted as the Subspace-Projection Method in the remainder of this paper.

An alternate approach to exploit the difference co-array has been developed by Pal and Vaidyanathan and is known as Rank Enhanced Spatial Smoothing (RESS). This algorithm exploits the hole-free co-array section of the co-prime array [6, 27]. In these works, they have shown how to develop a positive- 
Stage 3: Vectorize DDC

$$
\mathbf{y}=\operatorname{vec}\left(\mathbf{R}_{\mathbf{x}^{\prime}(t)}\right)=\mathbf{B} \mathbf{p}+\sigma_{n}^{2} \hat{\mathbf{e}}
$$

Where $\mathbf{B}$ is a ${Q^{\prime}}^{2} \times K$ complex matrix and each column of $\mathbf{B}$ is the Kronecker product between array manifold vectors of the sparse array and their conjugates. Namely, B can be written as,

$$
\mathbf{B}=\left[\mathbf{v}_{s}^{*}\left(\theta_{1}\right) \otimes \mathbf{v}_{s}\left(\theta_{1}\right), \cdots, \mathbf{v}_{s}^{*}\left(\theta_{K}\right) \otimes \mathbf{v}_{s}\left(\theta_{K}\right)\right]
$$

definite spatial covariance matrix for source localization from the array snapshot vector. The RESS algorithm can be applied to other array geometries that have contigous sections of hole-free co-array. In addition this technique can be applied to array geometries that produce hole-free difference co-arrays over the entire aperture of the array. Shakeri et al. have applied the RESS algorithm to Sparse Ruler Array designs for the localization of more sources than sensors 28. The development of the RESS algorithm is based on work of Ma et.al,

The RESS algorithm for the minimum temporal coherence period synthetic array can be described in the following major stages:

Stage 1: Co-array

The co-array is hole-free for spatial lags in the range, $0<l<(2 N-1) M+\eta_{0}$. This corresponds to an underlying ULA with $J=(2 N-1) M+\eta_{0}+1$ sensor elements.

Stage 2: Direct Data Covariance (DDC)

$$
\mathbf{R}_{\mathbf{x}^{\prime}(t)}=\mathbf{x}^{\prime}(t) \mathbf{x}^{\prime H}(t)=\sum_{i=1}^{K} \mathbf{v}_{s}\left(\theta_{i}\right) \mathbf{v}_{s}^{H}\left(\theta_{i}\right) \sigma_{i}^{2}+\sigma_{n}^{2} \mathbf{I}
$$

with $\mathbf{p}=\left[\sigma_{1}^{2}, \cdots, \sigma_{K}^{2}\right]^{\top}, \hat{\mathbf{e}}=\left[\mathbf{e}_{1}^{\top}, \mathbf{e}_{2}^{\top}, \cdots, \mathbf{e}_{Q^{\prime}}^{\top}\right]$, and $\mathbf{e}_{i}$ a vector of all zeros except for the $i$-th element.

Stage 4: $(2 J-1)$-ULA 
Each column of $\mathbf{B}$ has $2 J-1$ distinct values. These distinct values can be used to form the spatial covariances from a uniform linear array with $2 J-1$ sensors. The longer ULA is developed by extracting the distinct rows of $\mathbf{B}$, this results in a new matrix $\mathbf{B}_{1}$ with dimensions $(2 J-1) \times K$. The columns of $\mathbf{B}_{\mathbf{1}}$ form the array manifold vectors for a uniform line array with $2 J-1$ sensors with a phase center at the $J$-th sensor. The array snapshot for the $(2 J-1)$-ULA is,

$$
\mathbf{y}_{1}=\mathbf{B}_{1} \mathbf{p}+\sigma_{n}^{2} \mathbf{e}^{\prime} \in \mathbf{C}^{(2 J-1) \times 1}
$$

with $\mathbf{e}^{\prime}$ is a vector of all zeros except for a 1 at the $J$-th position.

\section{Stage 5: J-ULA Subarray}

The $(2 J-1)$-ULA we can subdivide into overlapping $J$-ULA subarrays. The elements of each subarray are taken from the larger ULA as, $\left\{(-i+1+n) \frac{\lambda}{2}\right\}_{n=0}^{J-1}$. The $J$-ULA subarray snapshot can be written as,

$$
\mathbf{y}_{1, i}=\mathbf{B}_{1 i} \mathbf{p}+\sigma_{n}^{2} \mathbf{e}_{i}^{\prime}
$$

where $\mathbf{B}_{1 i}$ is the $J \times K$ array manifold vector matrix for the $i$-th subarray and $\mathbf{e}_{i}^{\prime}$ is a zeros vector except for a 1 is the $i$-th position. For each subarray we can compute the $J \times J$ Rank-1 spatial covariance matrix as, $\mathbf{R}_{1 i}=\mathbf{y}_{1, i} \mathbf{y}_{1, i}^{H}$.

Stage 6: Rank Enhancement

$$
\mathbf{R}_{s \mathbf{x}^{\prime}(t)}=\frac{1}{J} \sum_{1=1}^{J} \mathbf{R}_{1 i}
$$

Theorem 1 in [27] proves that $\mathbf{R}_{s \mathbf{x}^{\prime}(t)}$ has the same form, but with squared eigenvalues, as the positive-definite spatial covariance matrix of a ULA with $J$ sensors. This matrix provides access to the degrees of freedom on the order of the synthetic array aperture. In addition, Liu and Vaidyanathan have developed a lower complexity method for co-array based processing. This method produces a covariance matrix estimate that can be used for source localization while relaxing the need for spatial smoothing in a signal/noise subspace based source localization technique [31. In the following section we analyze the source localization performance of DAA, Subspace projection, and RESS spatial covariance estimation methods. 


\section{Source Localization Simulation Results}

In this section, we explore the use of the co-prime array and SA processing for source localization. We consider the co-prime $(N=3, M=5)$ sensor array that consists of a total of 10 physical sensors spanning a $25 \frac{\lambda}{2}(\mathrm{~m})$ aperture. To enable co-array processing this array requires $1 \tau$ augmentation step, where $\tau$ is the time required to travel a total distance of $\lambda_{0} / 2(\mathrm{~m})$. For the simulations presented here, the wave propagation speed, array velocity, and source frequency $f_{0}$ are chosen to be $1500 \frac{\mathrm{m}}{\mathrm{s}}, 1.3 \frac{\mathrm{m}}{\mathrm{s}}$, and $440 \mathrm{~Hz}$, respectively.

In particular, we examine the Direct Augmentation Algorithm (DAA), Subspace Projection, and Rank Enhanced Spatial Smoothing (RESS) techniques for spatial covariance estimation. Our objective is to gain insights into these approaches and contrast these covariance matrix technique under a common wave-field. In addition, we consider the performance of each covariance matrix estimation technique over SNR and number of temporal array snapshots used in the covariance estimation process.

\subsection{Field-Directionality Mapping}

To evaluate the source localization performance of the co-prime array with SA processing we examine the spatial spectrum derived form the above spatial covariance matrix estimation techniques. We consider two different approaches to accomplish this task. The first approach is to use beamforming to compute the received power over a discrete set of angles $\theta$ across the field of view of the array. From this set of power readings we are able to detect sources in the far-field of the array. The second approach is to apply a well know algorithm for source localization, the MUSIC algorithm. MUSIC is the acronym for Multiple Signal Classification and is a high-resolution method based on the signal/noise subspace projections.

In both of the above approaches, we will hold the convention of scanning the array relative to broadside where angles are measured from the center of the aperture on the range of $\theta \in\left[-90^{\circ}, 90^{\circ}\right]$. 
The first approach for field-directionality mapping is obtained by computing the power received at the synthetic array from angle $\theta$. The mathematical model for this approach is given by,

$$
P(\theta)=\frac{1}{Q^{\prime 2}} \mathbf{V}(\theta)^{H} \hat{\mathbf{R}}_{a} \mathbf{V}(\theta)
$$

where $\mathbf{V}(\theta)$ is the synthetic array steering vector given by,

$$
\mathbf{V}(\theta)=\left[1, \exp \left(-j \Omega_{0} \frac{d_{1} \sin (\theta)}{c}\right), \cdots, \exp \left(-j \Omega_{0} \frac{d_{Q^{\prime}-1} \sin (\theta)}{c}\right)\right]^{\top}
$$

and $\hat{\mathbf{R}}_{a}$ is the spatial covariance matrix estimate from the synthetic array computed via one of the above described estimation methods. This method produces what is commonly referred to as the Spatial Power Spectrum.

The second approach is the well known Multiple Signal Classification algorithm (MUSIC), and is a subspace method where the spatial covariance matrix estimate, $\hat{\mathbf{R}}_{a}$, is decomposed into its signal and noise subspaces. Here

$$
\hat{\mathbf{R}}_{a}=\mathbf{U} \boldsymbol{\Lambda} \mathbf{U}^{-1}
$$

where $\mathbf{U}$ is a matrix of the eigenvectors and $\boldsymbol{\Lambda}$ is a diagonal matrix of the corresponding eigenvalues of $\hat{\mathbf{R}}_{a}$. The MUSIC spectrum is computed by evaluating the matrix polynomial

$$
f(\theta)=\frac{1}{\mathbf{V}(\theta)^{H} \hat{\mathbf{R}}_{a, \text { noise }} \mathbf{V}(\theta)}
$$

where $\mathbf{V}(\theta)$ is as defined above, and $\hat{\mathbf{R}}_{a, \text { noise }}$ represents the noise subspace of the spatial covariance matrix. The dimension of the noise subspace is a parameter that needs to be estimated and is typically taken to be the number of the smallest eigenvalues with approximately the same power. Here the idea is to evaluate the polynomial $\mathbf{V}(\theta)^{H} \hat{\mathbf{R}}_{a, \text { noise }} \mathbf{V}(\theta)$ and if this quantity is small that the steering vector $\mathbf{V}(\theta)$ lies in the compliment of the noise subspace. From this perspective one would say that $\mathbf{V}(\theta)$ lies in the signal subspace and therefore represents an angle at which a signal is present. For a more complete development of the MUSIC algorithm see [30]. 
Table 3: Covariance Matrix Estimation Summary

\begin{tabular}{|c|c|c|}
\hline Technique & Summary & $\begin{array}{l}\text { Algorithm } \\
\text { Parameters }\end{array}$ \\
\hline RESS & $\begin{array}{l}\text { Spatial smoothing technique ap- } \\
\text { plied to the hole-free component } \\
\text { of the difference co-array }\end{array}$ & None \\
\hline Subspace Projection & $\begin{array}{l}\text { Estimate array channel data via } \\
\text { subspace minimization }\end{array}$ & $\begin{array}{l}\text { Regularization } \\
\text { parameter } \mu\end{array}$ \\
\hline DAA & $\begin{array}{l}\text { Form a Toeplitz matrix from spa- } \\
\text { tial covariance estimates }\end{array}$ & $\begin{array}{l}\text { Array snapshot } \\
\text { support }\end{array}$ \\
\hline
\end{tabular}

In the remainder of this section we will compare and contrast the performance of the three different covariance matrix estimation techniques presented in section 4. The common objective in the use of each of the different techniques is to form an estimate of a spatial covariance matrix that approximates what would have been obtained from a ULA system, but with fewer sensors. In this sense, we are interested in the source localization and side-lobe performance of each technique. Table 3 summarizes each technique covariance matrix estimation technique.

The Subspace Projection method used the single snapshot DAA covariance matrix approximation to produce an array snapshot where all post synthetic aperture missing channels of data are estimated. In this respect, the Subspace Projection method produces an array snapshot that has active channels at all $\lambda / 2$ spacings over the aperture of the array, $\hat{q}$ is of the same dimension as a ULA over the same aperture. The regularization parameter $\mu$ is a tuning parameter in the subspace-projection optimization process that has an impact on the level of agreement between the channels produced during the SA process and the same channels produced after Subspace Projection. Figure 4 shows the norm-squared error between $\hat{q}(t)$ and the corresponding ULA snapshot $\mathbf{x}_{\mathrm{ULA}}(t)$. In this sense, 
Table 4: Source Location Ground Truth

\begin{tabular}{|l||l|}
\hline Angle $\theta^{\circ}$ & $-60^{\circ},-50^{\circ},-40^{\circ},-30^{\circ},-20^{\circ},-10^{\circ},-2.5^{\circ}$ \\
& $0^{\circ}, 5^{\circ}, 10^{\circ}, 20^{\circ}, 30^{\circ}, 40^{\circ}, 50^{\circ}, 60^{\circ}$ \\
\hline
\end{tabular}

we obtain an estimate of how close the output of the optimization process is to producing accurate channel data on any missing channels after the SA process is complete. The impact of $\mu$ on the Average norm-squared error can be seen in Figure 4 (a-b). Here the data suggests that for $10^{-4}<\mu<10^{-2}$ the mean error is minimum as well as has a minimum variance.

The DAA covariance matrix estimation method is sensitive to the number of snapshot used to average spatial covariance estimates. Figure 5 shows the eigenvalue spectra for a single source scenario with SNR of 0 (dB). Here we see that we require at least 55 array snapshots to produce non-negative eigenvalues.

Using this understanding of the effect of snapshot support for the DAA estimation technique and the effect of $\mu$ on the snapshot estimate for the Subspace Projection technique, we compare the source localization performance across the different techniques.

\subsection{Multiple Source Localization}

Consider the wave-field that is formed by 15 far-field sources, where the source angles relative to array broadside with an SNR of $0 \mathrm{~dB}$ are given in Table 4. Here sources are spaced uniformly over the intervals of $\left[-60^{\circ},-10^{\circ}\right]$ and $\left[10^{\circ}, 60^{\circ}\right]$ at increments of $10^{\circ}$. However, within the interval $\left(-10^{\circ}, 10^{\circ}\right)$ sources are spaced non-uniformly at $-2.5^{\circ}, 0^{\circ}$, and $5^{\circ}$.

Figure 6 shows the Spatial Power Spectrum and MUSIC Spectrum derived from each of the spatial covariance estimation methods. A total of 500 array snapshots are used to estimate the spatial covariance matrix for each estimation method and the SNR of each source is constant at $0(\mathrm{~dB})$. In these plots we compare the spatial spectrums of each covariance estimation method against that of the ULA. Figure 6(a) shows the Spatial Power Spectrum, in this plot we 
see that the spectrum derived from the RESS and DAA approximation methods are in good agreement with the ULA. In particular, these methods produce side-lobe levels equivalent to a ULA. However, the Subspace Projection methods suffers from higher side-lobes. The side-lobes of the Subspace Projection method are a result of the agreement between the estimated channel data produce by the choice of regularization parameter $\mu$. Here $\mu$ was chosen to minimize the error in the estimated channel data when compared to the ULA. Figure 6(a) also shows the Spatial Power Spectrum from the physical co-prime array with no SA processing. In this case, the side-lobes are the highest due to the nonuniformity of the sensor placement within the array. For each of the arrays and estimation methods the source at $-2.5^{\circ}$ and $0^{\circ}$ are not resolvable. Figure 6(b) shows the MUSIC spectrum derived form each estimation technique and the true ULA. Here in all but the Subspace Projection Method we are able to resolve all 15 sources.

\subsection{Performance Analysis}

We consider a wave-field with a single far-field source located at $0^{\circ}$ relative to array broadside. Here we perform Monte Carlo (MC) analysis over the number of snapshots used to estimate a given spatial covariance matrix and the SNR of the far-field source. In addition, we show the Mean and Variance of the Peak Side-lobe over both the number of snapshots and SNR parameters. The peak side-lobe informs the ability of the covariance matrix estimation techniques have to approach that of the ULA. A total of 100 noise realizations are used for each MC analysis.

Figure 7 shows the MC results over the number of array snapshots used to estimate a given covariance matrix. The RMSE of the bearing estimate are in good agreement between each of the covariance matrix estimation methods. However, the Peak Side-lobe level reveals performance differences in each estimation technique. Figure 7 (b-c) shows the mean and variance of the peak side-lobe level over a range of array snapshots. Here we see that as the number of snapshots increases the side-lobes from the DAA and RESS covariance estimates follow 
the trend of the ULA while the side-lobes of the Subspace Projection method do not. This discrepancy is the result of the choice of regularization parameter $\mu$, which controls the level of agreement between the estimated channel data. This limiting peak side-lobe level of the Subspace Projection method is the result of mismatch over the channel data.

Figure 8 shows the MC results over SNR. Here we see again that the RMSE of the bearing estimate are in good agreement with that of the ULA over the SNR range. However the Average Peak side-lobe level for both the RESS and DAA covariance matrix estimation methods follow that of the ULA. Again the effect of $\mu$ is seen in the average peak side-lobe measurement from the Subspace Projection method.

The above performance analysis suggest that the DAA and RESS covariance matrix estimation methods are capable of tracking the performance of the ULA, while the sensor array where these covariance matrix estimate are derived from contains fewer sensors. These simulation results suggest that while the Subspace Projection can produce accurate bearing estimates, the limiting factor in its performance is the Peak Side-lobe level.

\subsection{Synthetic Aperture Temporal Sampling Perturbation}

The synthetic aperture process presented in this work is centered on temporally sampling at desired spatial locations such that array snapshots from different time instants can be fused together to form a denser array. The synthetic array snapshot referenced at time $t$ is formed by fusing together sensor array snapshots from sample times $t+\tau, t+2 \tau, \cdots, t+\eta \tau$, where $\eta$ is the number of synthesis steps required to fill the difference co-array of the synthetic sensor array. Recall that $\tau$ is the time required for the array to travel a distance of $\lambda_{0} / 2(\mathrm{~m})$ for a given velocity $v \mathrm{~m} / \mathrm{s}$. The process of forming virtual sensors amounts to developing desired phase delays from the Doppler-shift and physical array manifold vector to account for virtual sensors at desired spatial locations. Recall that this is expressed mathematically from equation 8 and 9 of section 2 . Here we see that the $l$-th segment of elements of the synthetic array manifold 
vector, $\tilde{\mathbf{b}}_{l}\left(\theta_{k} ; \Omega_{0}\right)$ where $l \in\{1,2, \cdots, \eta\}$, is given by,

$$
\tilde{\mathbf{b}}_{l}\left(\theta_{k} ; \Omega_{0}\right)=\exp \left(-j \Omega_{0} v l \tau \frac{\sin \left(\theta_{k}\right)}{c}\right) \mathbf{b}\left(\theta_{k} ; \Omega_{0}\right)
$$

where $\mathbf{b}\left(\theta_{k} ; \Omega_{0}\right)=\left[1, \exp \left(-j \Omega_{0} \frac{d_{1} \sin \left(\theta_{k}\right)}{c}\right), \cdots, \exp \left(-j \Omega_{0} \frac{d_{Q-1} \sin \left(\theta_{k}\right)}{c}\right)\right]^{\top}$ is the array manifold vector of the physical array and $\tau$ is the sampling instant such that, $v \tau=\lambda_{0} / 2(\mathrm{~m})$.

To examine the robustness of the synthetic aperture sampling process we consider the effects of a random offset in half-wavelength sampling instant $\tau$. Let the modified sampling instant $\tau^{\prime}=\tau+\delta$, where $\delta \sim \mathcal{N}\left(0, \sigma_{\delta}^{2}\right)$ and $\delta$ carries the units of seconds. With this model we see that virtual sensors are no longer realized at half-wavelength intervals instead the virtual sensor locations are normally distributed $\lambda_{0} / 2+v \delta \sim \mathcal{N}\left(\frac{\lambda}{2}, v^{2} \sigma_{\delta}^{2}\right)$ random variables.

In order to evaluate the effect of the random offset in half-wavelength sampling instant we consider the situation of estimating a spatial covariance matrix using the RESS algorithm compared to a ULA with no synthetic aperture processing. The evaluation metric is the angle $\phi$ between the signal subspaces derived from each spatial covariance matrix and is given by,

$$
\phi\left(V_{1}, V_{2}\right)=\cos \left(\left|V_{1} \top V_{2}\right|\right)^{-1}
$$

where an angle near zero implies the subspaces are nearly co-linear, while an angle $\frac{\pi}{2}$ indicates orthogonal subspaces. In particular, we measure the angle between the signal subspace derived from the RESS spatial covariance matrix with perturbed sampling realizations against the signal subspace derived from a ULA with no synthetic aperture processing.

In this simulation, the signal field is made of three far-field sources with SNR of $5 \mathrm{~dB}$ at arrival angles $-8^{\circ}, 10^{\circ}, 18^{\circ}$, referenced to array broadside. Each spatial covariance matrix is estimated from 200 array snapshots. Figure 7 shows the subspace matching performance as a function of offset variance $\sigma_{\delta}^{2}$. For each variance $\sigma_{\delta}^{2} 100$ Monte Carlo iterations of subspace measurement are computed. The average subspace angle and first standard deviation are shown for each offset 
variance. Figure 9 suggests that the synthetic aperture process in not robust to offsets in the sampling times used for creating virtual sensors at the desired spatial locations. Current work is underway to implement correction factors for timing offsets into the processing scheme and was not the focus of the work presented here. This includes applying time domain interpolation to compute the sensor data at the required time instants $t+\tau, t+2 \tau, \cdots, t+\eta \tau$. In addition, sampling errors can arise form array shape deformation due to sea conditions. Addressing this issue is beyond the scope of this work.

The key point in the experiments presented above is the performance of the co-prime array with minimal SA processing. The primary advantage of using the co-prime array in the SA setting is the minimum temporal coherence requirements in order to approximate the performance of a ULA in idealized signal environments. However, it remains to develop an effective difference co-array SA methods to deal with offsets in sampling as well more complicated signal models. This is a topic of current work that will be developed in upcoming publications.

\section{Conclusion}

In this work, we considered the use of synthetic aperture processing for improving the performance of a thinned array system. In particular the primary objective was to use a thinned array to approximate the side-lobe level and source localization performance of a ULA. The fundamental trade-off between this approach and processing data with a true ULA is computational complexity. This complexity is derived from the SA processing along with the spatial covariance matrix estimation requirements. However, in situations where resources are limited or sensors fail, in moving array systems, these approaches can be applied to mitigate performance losses. The primary focus of the application of $\mathrm{SA}$ in this work is to address the limitations in the spatial covariance lag estimation range of linear co-prime arrays. Co-prime arrays are a particular class of thinned arrays that have spatial covariance lag coverage guarantees that make 
them attractive candidates for SA processing. In the static setting co-prime arrays provide a contiguous range of spatial covariances of $\mathcal{O}(M N)$ while the array has only $\mathcal{O}(M+N)$ sensors. In addition, this property of co-prime arrays scales with co-prime factors $M, N$ allowing for large aperture arrays to be easily designed. This characteristic of co-prime arrays is what makes them appealing in sensing applications.

In particular, the goal of this work is to maximize the spatial covariance sensing capability of the co-prime array system, while minimizing the synthetic aperture processing time requirements. We have shown that the minimum number of SA iterations in order to produce a hole-free co-array is a function of the co-prime parameters $N, M$ and is approximately half of the smaller of the two parameters. This implies that spatial coherence of the environment is required for the amount of time required for the array to traverse only a fraction of total array aperture. In addition, we have presented three methods for computing spatial covariance matrices from the SA array snapshots. These methods varied in approach and computational complexity. While each method is suitable under certain conditions, they each are able to produce reliable covariance matrix estimates. In principle, these techniques can be applied to other thinned array geometries and alternative minimum coherence time requirements can be developed.

Overall, SA processing was shown to improve the array processing performance of the co-prime array. By virtue of adjoining virtual sensors at halfwavelength spacings, we are able to reduce the peak side-lobe level of the physical array. However, the true performance gain comes from the ULA equivalence derived from the spatial covariance estimates acquired from the synthetic array snapshot. While the synthetic array remains a thinned array, the corresponding difference co-array is hole-free. This property enables processing techniques the exploit the spatial covariance lag coverage of the synthetic array. To this end we have shown that it is possible to approximate the side-lobe level of a ULA from synthetic array. However, when ideal conditions are relaxed these properties quickly deteriorate. The focus of future work on the subject is to develop and 
investigate strategies for accounting for mismatch in SA processing and allowing for more realistic signal environments.

\section{Acknowledgment}

This work was supported by the Office of Naval Research (ONR) Code 321US grant no. N00014-13-1020-1.

\section{References}

[1] R. L. Haupt, Thinned arrays using genetic algorithms, Antennas and Propagation, IEEE Transactions on 42 (7) (1994) 993-999. doi:10.1109/8. 299602

[2] S. Holm, Sparse and irregular sampling in array processing, in: Acoustics, Speech, and Signal Processing, 2000. ICASSP '00. Proceedings. 2000 IEEE

International Conference on, Vol. 6, 2000, pp. 3850-3853 vol.6. doi:10. 1109/ICASSP. 2000.860243.

[3] H. Oraizi, F. Fallahpout, Nonuniformly spaced linear array design for the specified beamwidth/sidelobe level or specified directivity/sidelobe level with coupling consideration, in: Progress In Electromagnetics Research, 2008.

[4] A. Moffet, Minimum-redundancy linear arrays, Antennas and Propagation, IEEE Transactions on 16 (2) (1968) 172-175.

[5] K. Blanton, J. McClellan, New search algorithm for minimum redundancy linear arrays, in: Acoustics, Speech, and Signal Processing, 1991. ICASSP91., 1991 International Conference on, 1991, pp. 1361-1364 vol.2. doi: 10.1109/ICASSP.1991.150675.

[6] P. Pal, P. Vaidyanathan, Nested arrays: A novel approach to array processing with enhanced degrees of freedom, IEEE Trans. Signal Process. 58 (8) (2010) 4167-4181. doi:10.1109/TSP.2010.2049264. 
[7] P. Vaidyanathan, P. Pal, Sparse sensing with co-prime samplers and arrays, IEEE Trans. Signal Process. 59 (2) (2011) 573-586. doi:10.1109/TSP. 2010.2089682

[8] E. BouDaher, Y. Jia, F. Ahmad, M. G. Amin, Multi-frequency co-prime arrays for high-resolution direction-of-arrival estimation, IEEE Transactions on Signal Processing 63 (14) (2015) 3797-3808. doi:10.1109/TSP. 2015. 2432734 .

[9] M. Johnson, Synthetic elements for moving line arrays, in: OCEANS 2009 - EUROPE, 2009, pp. 1-6. doi:10.1109/OCEANSE.2009.5278113

[10] M. H. Johnson, Method for mitigating spatial aliasing (09 2011).

[11] S. Pillai, F. Haber, Statistical analysis of a high resolution spatial spectrum estimator utilizing an augmented covariance matrix, Acoustics, Speech and Signal Processing, IEEE Transactions on 35 (11) (1987) 1517-1523. doi: 10.1109/TASSP. 1987.1165068

[12] Y. Abramovich, D. Gray, A. Gorokhov, N. Spencer, Positive-definite toeplitz completion in doa estimation for nonuniform linear antenna arrays. i. fully augmentable arrays, IEEE Trans. Signal Process. 46 (9) (1998) 2458-2471. doi:10.1109/78.709534

[13] Y. Nai-Chyuan, C. William, Application of synthetic aperture processing to towed array data, J. Acoust. Soc. A. 86 (2) (1989) 754-765. doi:http://dx.doi.org/10.1121/1.398758.

11 URL http://scitation.aip.org/content/asa/journal/jasa/86/2/ $10.1121 / 1.398758$

[14] R. Williams, B. Harris, Passive acoustic synthetic aperture processing techniques, IEEE J. Ocean. Eng. 17 (1) (1992) 8-15. doi:10.1109/48.126949

[15] S. Stergiopoulos, H. Urban, A new passive synthetic aperture technique for towed arrays, Oceanic Engineering, IEEE Journal of 17 (1) (1992) 16-25. doi:10.1109/48.126950. 
[16] F. Haber, M. Zoltowski, Spatial spectrum estimation in a coherent signal environment using an array in motion, IEEE Trans. Antennas Propag. 34 (3) (1986) 301-310. doi:10.1109/TAP.1986.1143831

[17] Y. Zuo, G. Li, J. Xu, Y.-N. Peng, X.-G. Xia, Detection and location of fast moving targets using minimum redundancy linear array sar, in: Radar, 2006. CIE '06. International Conference on, 2006, pp. 1-4. doi:10.1109/ ICR.2006.343306.

[18] L. Bo, S. Chao, A new method for array extension based on minimum redundancy linear array, in: Signal Processing (ICSP), 2010 IEEE 10th In-

ternational Conference on, 2010, pp. 332-335. doi:10.1109/ICOSP.2010. 5654981

[19] J. Ramirez, J. Odom, J. Krolik, Exploiting array motion for augmentation of co-prime arrays, in: Sensor Array and Multichannel Signal Processing Workshop (SAM), 2014 IEEE 8th, 2014, pp. 525-528. doi:10.1109/SAM. 2014.6882458

[20] J. Ramirez, J. Krolik, Multiple source localization with moving co-prime arrays, in: Acoustics, Speech and Signal Processing (ICASSP), 2015 IEEE International Conference on, 2015.

[21] S. Stergiopoulos, E. J. Sullivan, Extended towed array processing by an overlap correlator, J. Acoust. Soc. A. 86 (1).

[22] R. Hoctor, S. Kassam, The unifying role of the coarray in aperture synthesis for coherent and incoherent imaging, Proceedings of the IEEE 78 (4) (1990) 735-752. doi:10.1109/5.54811.

[23] R. E. Williams, H. F. Battestin, Time coherence of acoustic signals transmitted over resolved paths in the deep ocean, The Journal of the Acoustical Society of America 59 (2).

[24] R. E. Williams, Creating an acoustic synthetic aperture in the ocean, The Journal of the Acoustical Society of America 60 (1). 
[25] T. C. Yang, Measurements of temporal coherence of sound transmissions through shallow water, The Journal of the Acoustical Society of America $120(5)$.

[26] O. Kazanci, J. Krolik, Single-snapshot beamformer performance using large arrays with faulty sensors, Sensor Array and Multichannel Processing, 2006. Fourth IEEE Workshop on (2006) 6-10doi:10.1109/SAM.2006.1706073.

[27] P. Pal, P. Vaidyanathan, Coprime sampling and the music algorithm, in: Digital Signal Processing Workshop and IEEE Signal Processing Education Workshop (DSP/SPE), 2011 IEEE, 2011, pp. 289-294. doi: 10.1109/DSP-SPE.2011.5739227.

[28] S. Shakeri, D. Ariananda, G. Leus, Direction of arrival estimation using sparse ruler array design, in: Signal Processing Advances in Wireless Communications (SPAWC), 2012 IEEE 13th International Workshop on, 2012, pp. 525-529. doi:10.1109/SPAWC.2012.6292964.

[29] W.-K. Ma, T.-H. Hsieh, C.-Y. Chi, Doa estimation of quasi-stationary signals via khatri-rao subspace, in: Acoustics, Speech and Signal Processing, 2009. ICASSP 2009. IEEE International Conference on, 2009, pp. 21652168. doi:10.1109/ICASSP. 2009.4960046.

[30] R. Schmidt, Multiple emitter location and signal parameter estimation, Antennas and Propagation, IEEE Transactions on 34 (3) (1986) 276-280. doi:10.1109/TAP.1986.1143830.

[31] C. L. Liu, P. P. Vaidyanathan, Remarks on the spatial smoothing step in coarray music, IEEE Signal Processing Letters 22 (9) (2015) 1438-1442. doi:10.1109/LSP. 2015.2409153. 


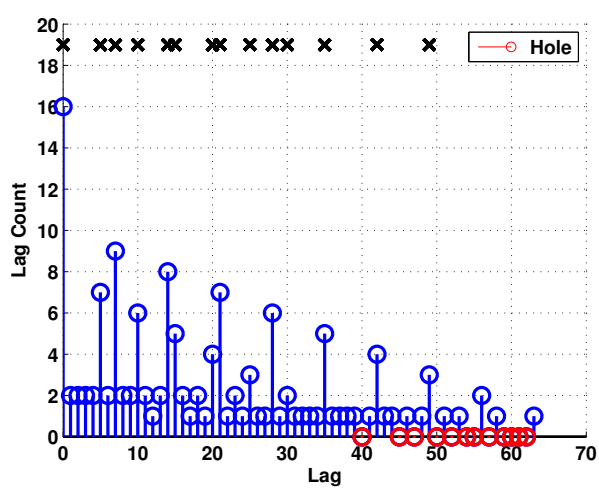

(a) Physical Array Difference Co-array

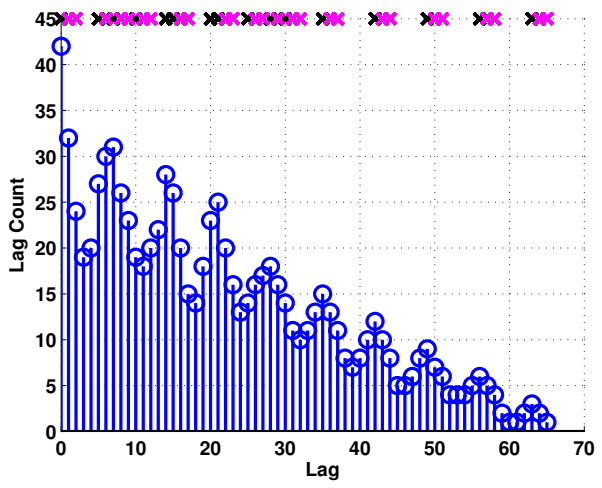

(b) Synthetic Array Difference Co-array, $\eta_{0}=2$

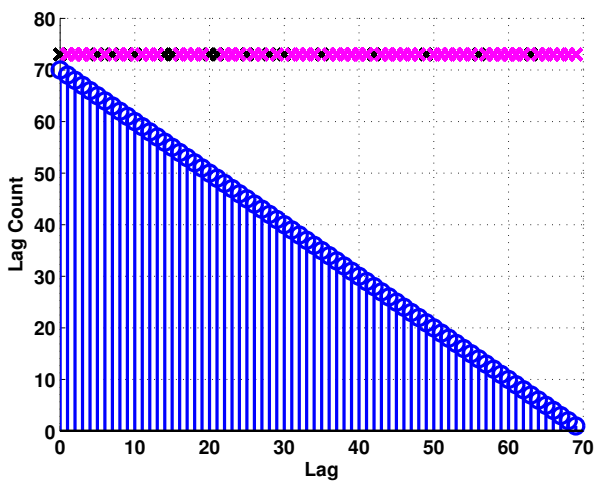

(c) Synthetic Array Difference Co-array, $\eta=6$

Figure 3: Difference Co-array for different stages of synthetic aperture processing. 


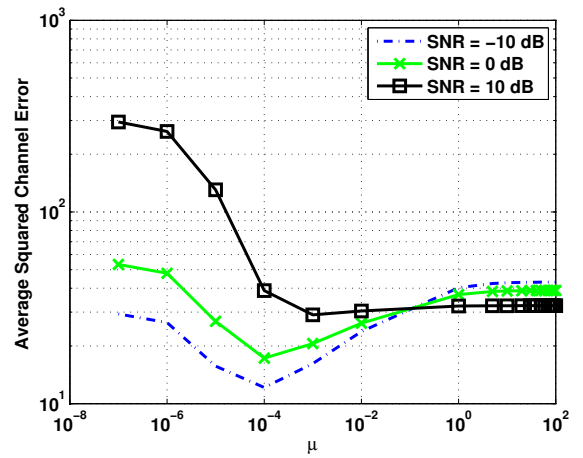

(a) Average Channel Error

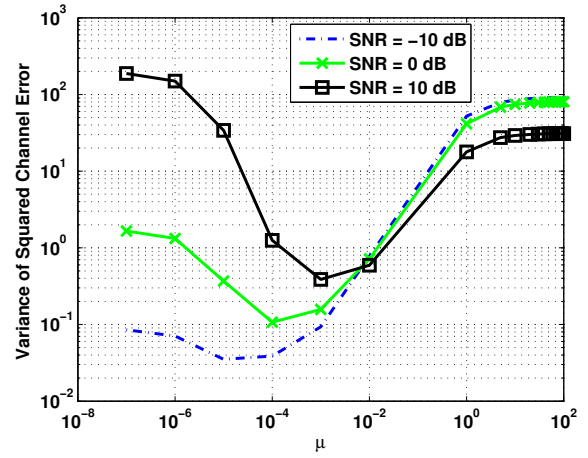

(b) Variance of Channel Error

Figure 4: Subspace Projection Channel Error 


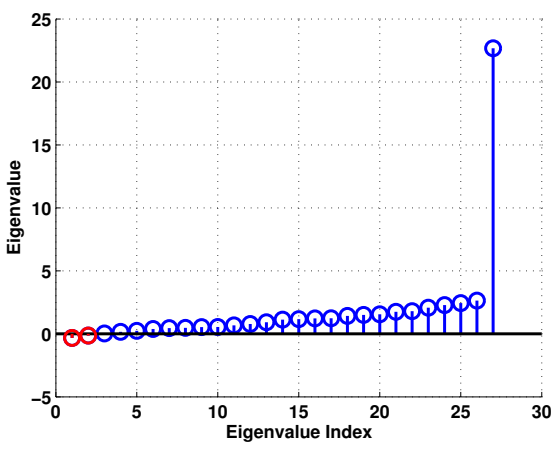

(a) 10 Array Snapshots

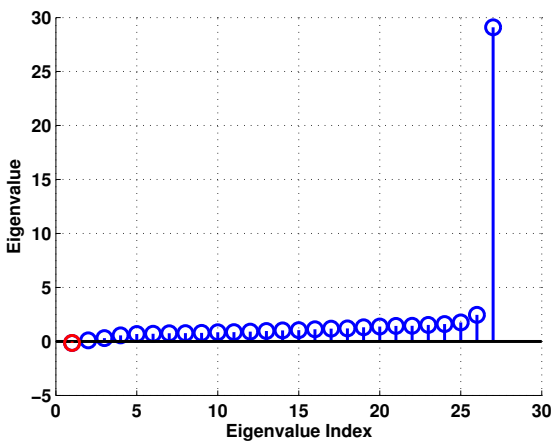

(b) 25 Array Snapshots

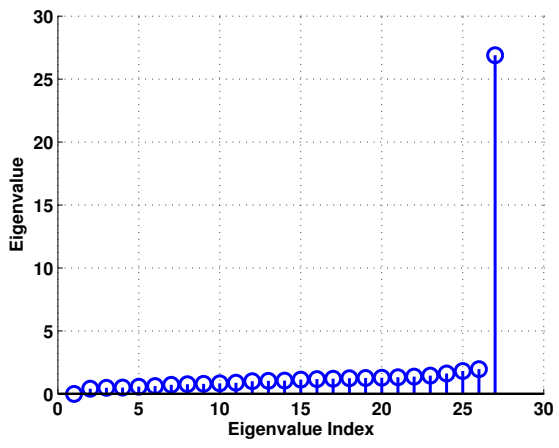

(c) 55 Array Snapshots

Figure 5: DAA Covariance Matrix Eigenvalues 


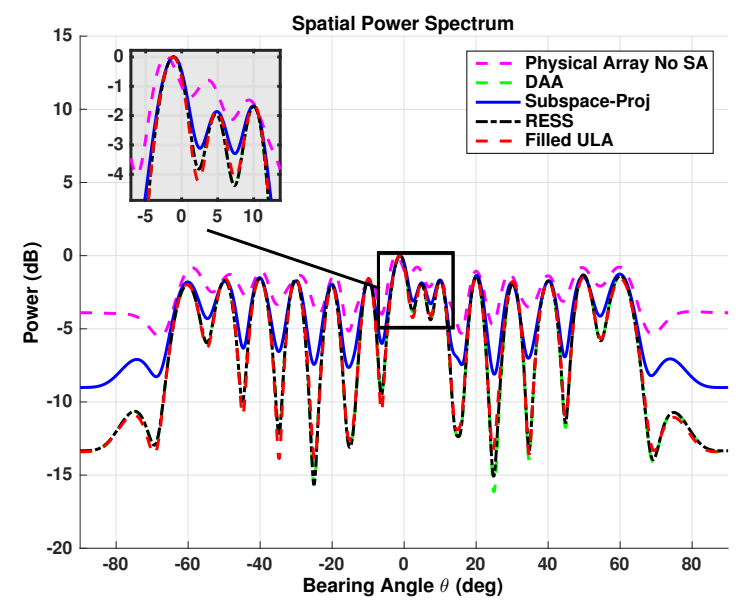

(a) Spatial Power Spectrum

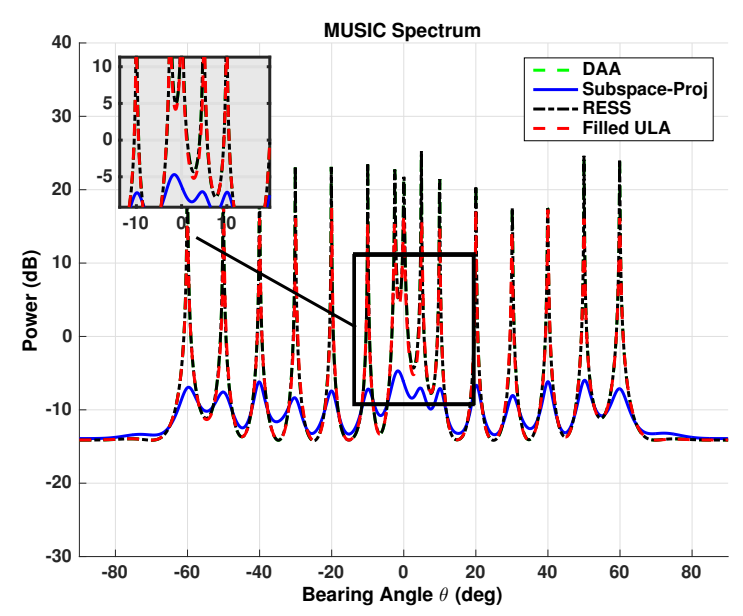

(b) MUSIC Spectrum

Figure 6: Source Localization 


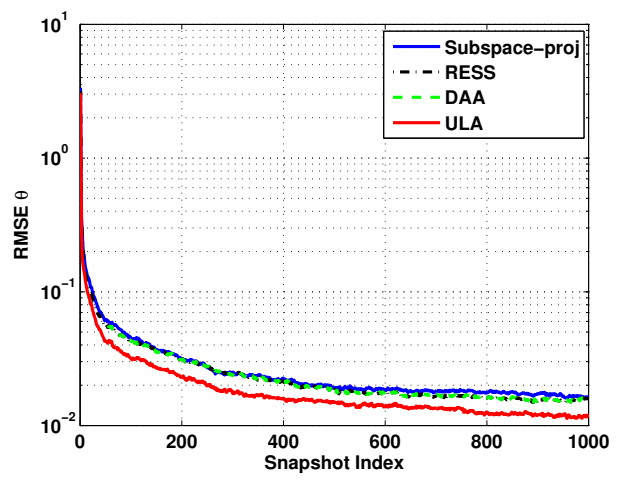

(a) Bearing RMSE

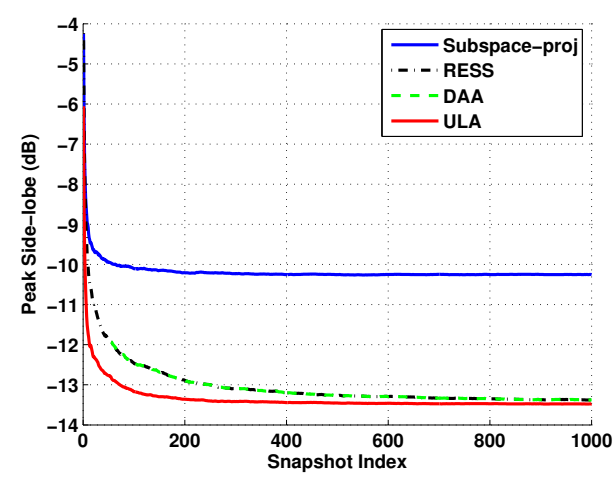

(b) Average Peak Side-lobe

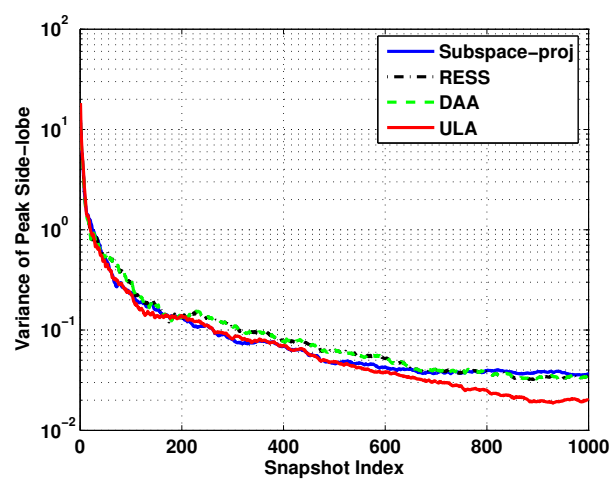

(c) Variance of Peak Side-lobe

Figure 7: Array Snapshot Monte Carlo 


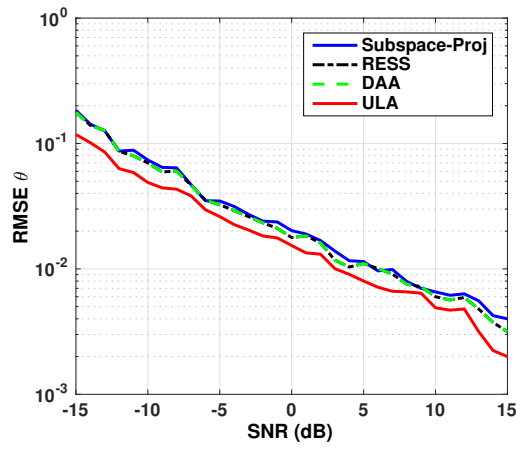

(a) Bearing RMSE

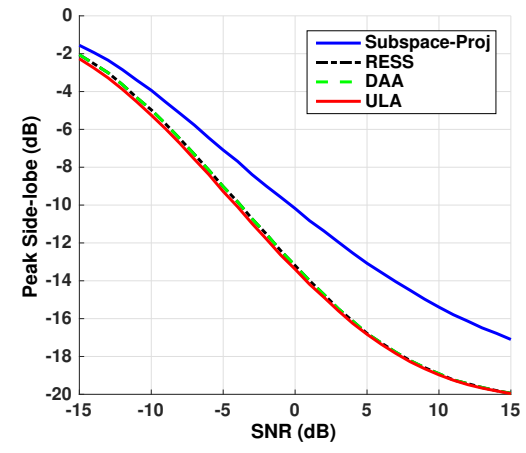

(b) Average Peak Side-lobe

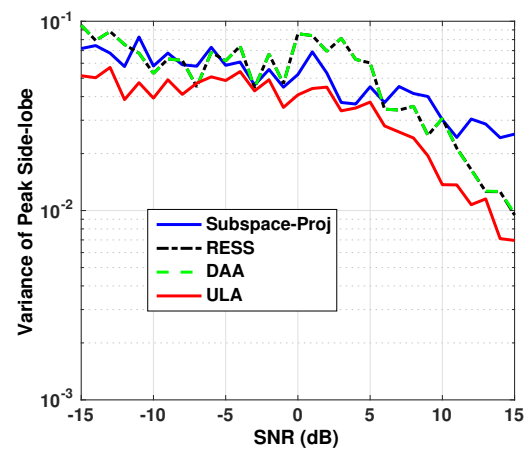

(c) Variance of Peak Side-lobe

Figure 8: SNR Monte Carlo 


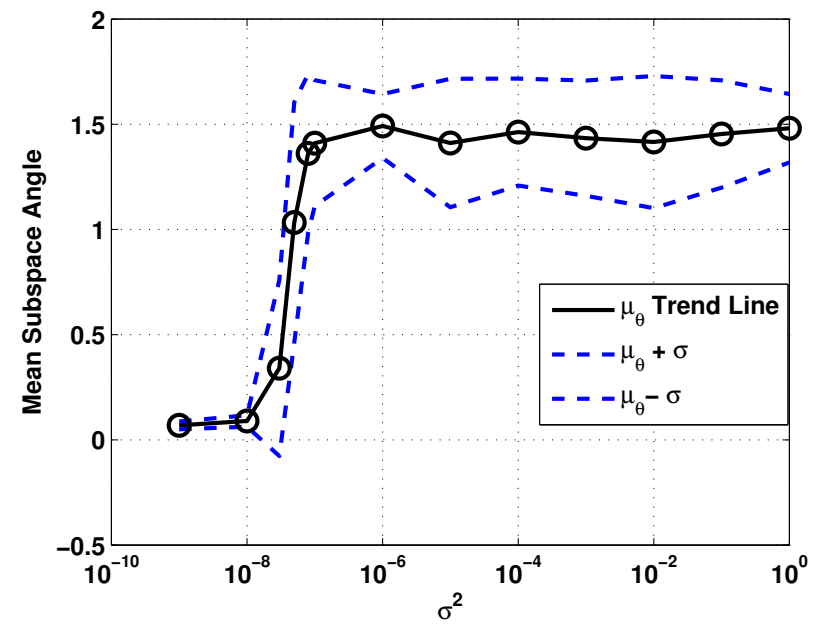

Figure 9: Subspace Angle (Rad) 